\title{
Agentes hepatoespecíficos, usos actuales: más allá de la caracterización de lesiones focales
}

\section{Hepatospecific Agents, Current Uses: More than the Characterization of Focal Lesions}
G. A. Castrillón ${ }^{1}$
J. P. Espinosa ${ }^{2}$
M. Noriega P. ${ }^{3}$
M. Royero 4
D. V. Gómez ${ }^{5}$

${ }^{1}$ Medico Radiólogo, Especialista en Imagen Corporal, Profesor Titular, Departamento de Radiología y Grupo de Gastrohepatología, Universidad de Antioquia, Medellín, Colombia

2 Radiólogo, CES, Fellow en Imagen Corporal, Universidad de Antioquia, Medellín, Colombia

${ }^{3}$ Mónica Noriega P., Residente de Radiología, Universidad de Antioquia, Medellín, Colombia

${ }^{4}$ Mónica Royero, Radióloga, Universidad de Antioquia, Medellín, Colombia

${ }^{5}$ Diana V Gómez, Medica Radióloga, Especialista en Imagen Corporal, Universidad de Antioquia, Medellín, Colombia

\author{
Address for correspondence German A. Castrillón, MD, \\ Departamento de Radiología y Grupo de Gastrohepatología, \\ Universidad de Antioquia, Medellín, Colombia \\ (e-mail: german.castrillon@udea.edu.co; \\ germanacastrillon@gmail.com).
}

\section{Resumen}

\section{Palabras Clave}

- hígado

- medio de contraste

- resonancia magnética
Con el ácido gadoxético, el realce dinámico de las lesiones focales es igual a los demás medios de contraste extracelulares, por lo que su utilidad principal está en la obtención de una fase hepatobiliar que permita diferenciar y caracterizar lesiones que contienen hepatocitos como en el caso de la hiperplasia nodular focal (HNF) y los nódulos regenerativos. Las lesiones que no tienen hepatocitos o que son carentes de conductos biliares, demuestran baja señal con respecto al parénquima hepático en esa fase, lo que ocurre con los adenomas hepatocelulares (AHC), nódulos displásicos de alto grado, carcinoma hepatocelular $(\mathrm{CHC})$ y metástasis entre otras. Hacer esta diferenciación cambia el manejo de la lesión focal, permitiendo en la mayoría de los casos caracterizar las lesiones que contienen hepatocitos que no requieren biopsia, como las benignas, de aquellas que si pudieran requerir la confirmación histológica. Otros usos de estos medios de contraste órgano específicos es la evaluación de la integridad de la vía biliar especialmente en pacientes posquirúrgicos y la diferenciación de quistes que tienen comunicación con la vía biliar de aquellos que son quistes hepáticos simples.

El objetivo de este artículo es realizar una revisión sobre la evidencia actual del papel emergente de los contrastes órgano específicos, incluyendo no solo la evaluación de las lesiones focales hepáticas, sino también otras utilidades como el análisis de la patología biliar. received

March 21, 2017

accepted

August 6, 2017
DOI https://doi.org/

$10.1055 / \mathrm{s}-0038-1637027$.

ISSN 1852-9992.
Copyright $\odot$ 2019, Sociedad Argentina de Radiología. Publicado por Thieme Revinter Publicações Ltda., Rio de Janeiro, Brazil. Todos los derechos reservados.
License terms

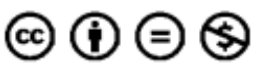




\begin{abstract}
With gadoxetic acid the dynamic enhancement of the lesions is equal to the other extracellular contrast agents. Its main utility is in obtaining a hepatobiliary phase that allows to characterize the lesions that contain functional hepatocytes as it would be the case of the regenerative nodules and focal nodular hyperplasia.

Lesions without functional hepatocytes or that are devoid of bile ducts appear hypointense with respect to the hepatic parenchyma at this stage, which occurs in adenoma, high-grade dysplastic nodules, hepatocarcinoma, and metastases among others. Making this differentiation, the management of the focal lesion would change, allowing us, in most cases, characterizing lesions containing functional hepatocytes as benign and therefore that dońt require biopsy, of those that may require histological confirmation.

Other uses of these contrast media are the evaluation of the integrity of the bile duct

Keywords

- liver

- contrast media

- magnetic resonance imaging

especially in post-surgical stages and the differentiation of cysts that have communication with the bile duct of those who are simple hepatic cysts.

The objective of this article is to perform a review of the current evidence about the emergent role of organ specific agents, not only for the evaluation of liver focal lesions, but also other utilities such as analysis of biliary pathology.
\end{abstract}

\section{Introducción}

Para la realización de la resonancia magnética (RM) hepatobiliar se utilizan dos tipos de medios de contraste, los extracelulares y los intracelulares. El primer grupo son los medios de contraste derivados del gadolinio que tienen un comportamiento intravascular y extracelular. ${ }^{1-5}$ El segundo grupo, conocidos también como órgano específicos, tiene un comportamiento intravascular, extracelular e intracelular. Actualmente, dos de estos agentes están disponibles comercialmente: Gadobenato de dimeglumina (Gd-BOPTA) y ácido gadoxético (Gd-EOB-DTPA) ${ }^{6-9}$ Ese último, por su disponibilidad en nuestro medio, será el enfoque de la presente revisión.

El ácido gadoxético es de gran utilidad en la detección y caracterización de lesiones focales hepáticas y en la valoración de la vía biliar, suministrando información adicional a las secuencias convencionales. ${ }^{10-17}$ Debido a su estructura molecular, que contiene una parte hidrosoluble $\mathrm{y}$ otra lipofílica (EOB-etoxibencil), muestra un mecanismo de acción bifásico. Inicialmente presenta una distribución en el espacio extracelular que permite la adquisición de imágenes dinámicas (arterial, portal y transicional). También presenta una distribución intracelular con captación selectiva por los hepatocitos y excreción hacia la vía biliar. Esta fase es conocida como hepatobiliar, y se obtiene aproximadamente a los 20 minutos luego de la inyección del medio de contraste. ${ }^{6,18}$ La captación se realiza a través de la proteína de membrana transportadora de aniones orgánicos (OATP1B y OATP1B3) y la excreción biliar sucede por medio de la proteína de resistencia multidroga (MRP2). Este proceso brinda información sobre la composición tisular de algunos tumores, la funcionalidad del hepatocito y la anatomía e integridad de la vía biliar $2,14,19-21$ (-Fig. 1).
La disfunción hepática y la obstrucción biliar pueden alterar la farmacocinética del contraste, retardando y disminuyendo la captación del medio de contraste por el hepatocito, lo que a su vez disminuye la intensidad de la señal hepática y su excreción en la fase hepatobiliar. ${ }^{22-24}$

Con respecto a la farmacocinética del ácido gadoxético, este compuesto tiene una vida media de una hora y es eliminado en similares proporciones por vía biliar en un $43-53 \%$ y renal en un $41-51 \%^{3,25-29}$ En lo referente al Gadobenato de dimeglumina (Gd-BOPTA), la fase

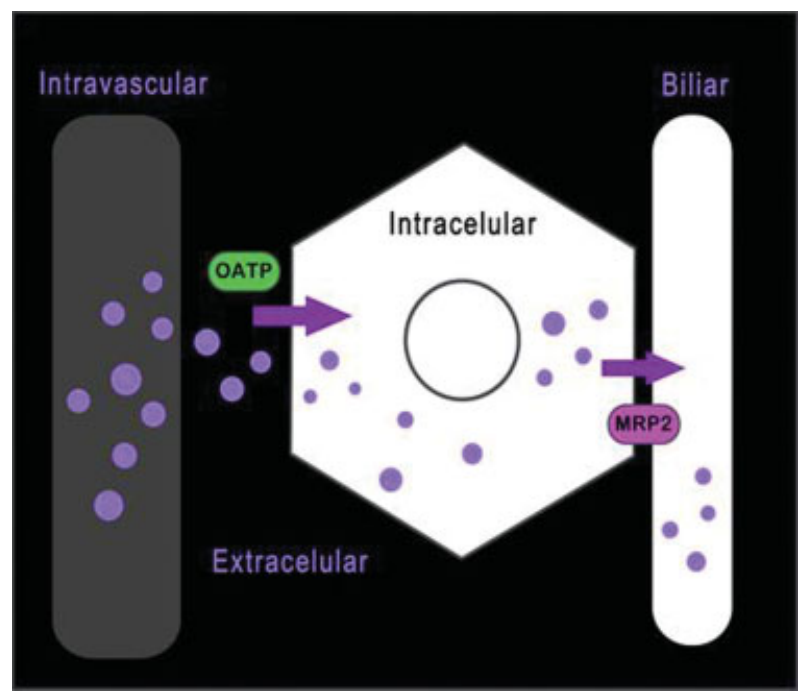

Fig. 1 Esquema del transporte del ácido gadoxético en el hepatocito. El contraste es captado activamente por el hepatocito a través del proteína de membrana transportadora de aniones orgánicos (OATP), lo cual permite información sobre la capacidad funcional del hígado; $y$ al ser excretados por la vía biliar mediante la proteína de resistencia multidroga (MRP2), evalúa la función y anatomía de esta. 
hepatobiliar se presenta típicamente entre los 45 y 120 minutos, su eliminación es 3-5\% biliar y tiene una vida media entre 1-2 horas. ${ }^{7,17}$

\section{Hallazgos por imagen}

Con el ácido gadoxético el realce dinámico de las lesiones es igual a los otros medios de contraste extracelulares. La dosis utilizada es de $0.025 \mathrm{mmol} / \mathrm{kg}$ de peso. Tanto las lesiones hipervasculares como los vasos sanguíneos pueden tener menor señal en la fase arterial comparado con los agentes extracelulares. $^{6}$

En la fase hepatocelular, el parénquima hepático aparece homogéneamente hiperintenso desde los 20 minutos hasta los 120 minutos luego de la administración del agente en las secuencias ponderadas en T1 a dosis de $0.025-0.050 \mathrm{mmol} /$ $\mathrm{kg}$. Adicionalmente el contraste se hace visible en los conductos biliares, y las estructuras vasculares aparecen hipointensas respecto al parénquima hepático ${ }^{30-32}$ (- Fig. 2).

La captación intralesional del ácido gadoxético en la fase hepatobiliar indica que la lesión contiene hepatocitos con las proteínas transportadoras específicas, como sería el caso de los nódulos regenerativos y la hiperplasia nodular focal (HNF). ${ }^{33,34}$ Las lesiones que no tienen hepatocitos funcionales o que son carentes de conductos biliares aparecen hipointensas respecto al parénquima hepático en esta fase, lo que ocurre en el caso de los adenomas hepatocelulares (AHC), los nódulos displásicos de alto grado, el carcinoma hepatocelular ( $\mathrm{CHC}$ ) y metástasis entre otras. 6

Las estructuras vasculares y los hemangiomas son de baja señal en la fase hepatobiliar, excepto cuando existe alteración de la función hepática por colestasis o daño hepatocelular. En esas circunstancias, el realce del parénquima puede ser débil, haciendo que las estructuras vasculares y los hemangiomas se observen isointensos en la fase hepatobiliar. ${ }^{6,11,35}$ Es así como, al usar medios de contraste órgano específicos, hay una mayor detección de las diferentes lesiones focales hepáticas ya que se comportan de acuerdo a su histología y se aumenta de $80 \%$ a $87 \%$ el rendimiento diagnóstico de la $\mathrm{RM}^{36,37}$

En la - Tabla 1 se resumen los hallazgos en la RM con medios de contraste órgano específicos de las diferentes lesiones focales.

\section{Utilidades y controversias}

La diferencia entre HNF y adenoma en la fase hepatobiliar consiste en que la HNF presenta una retención del contraste hasta en el $96 \%$ de los casos, a diferencia del adenoma que no lo retiene. ${ }^{10,27,36,38-40}$ Por lo tanto, el ácido gadoxético es útil para su diferenciación, con una sensibilidad entre $91-100 \%$ y una especificidad entre $87-100 \%{ }^{33}$ Sin embargo, algunos adenomas, principalmente los de tipo inflamatorio, pueden tener mínimo realce en esa fase. ${ }^{41}$

Con respecto a las lesiones metastásicas, el ácido gadoxético ha demostrado ser superior a otras modalidades por imagen para la detección de metástasis hepáticas especialmente en lesiones menores a $1 \mathrm{~cm} .{ }^{13,42-45}$ Tanto las metástasis hipervasculares como las hipovasculares no demuestran realce en la fase hepatobiliar. ${ }^{6}$

El CHC usualmente demuestra baja intensidad de señal en la fase hepatobiliar. El grado y homogeneidad de la hipointensidad es variable, dependiendo de la concentración y función de los transportadores, lo cual va ligado al grado de diferenciación histológica. ${ }^{2,3,20}$ Algunos CHC pueden tener realce paradójico (2.5-8.5\%), siendo la mayoría bien diferenciados con preservación de los sistemas trasportadores mencionados, lo cual favorece la acumulación de contraste intratumoral. ${ }^{12,20,46-48}$

Debido a su excreción biliar, el ácido gadoxético permite valorar la anatomía y la función de la vía biliar junto con las secuencias de colangioresonancia; de esta manera se pueden diferenciar lesiones biliares de las extra-biliares, evaluar
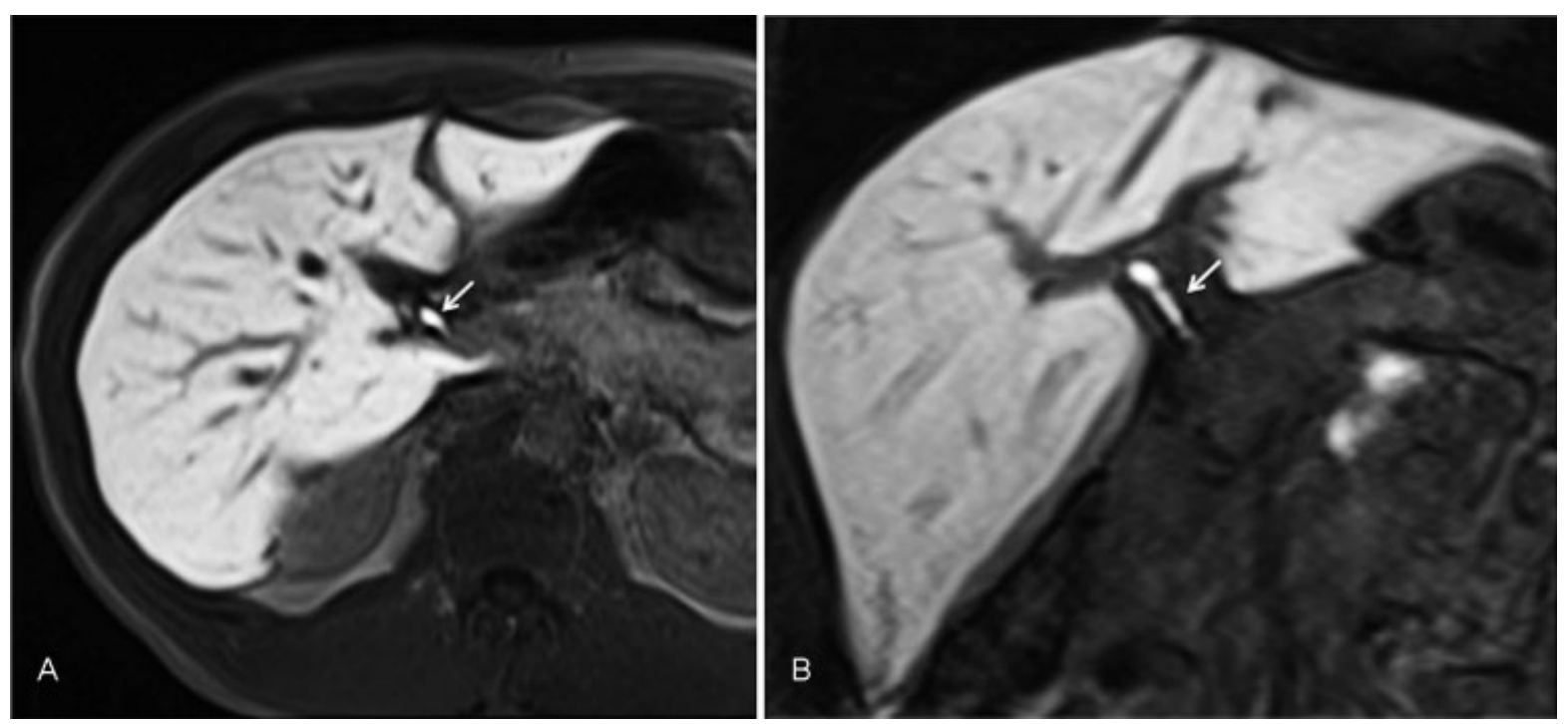

Fig. 2 Estudio de RM. Secuencia ponderada en T1 en fase hepatobiliar, axial (A) y coronal (B). Se observa hiperintensidad del parénquima hepático y de la vía biliar (flechas) por la excreción hepatobiliar. 


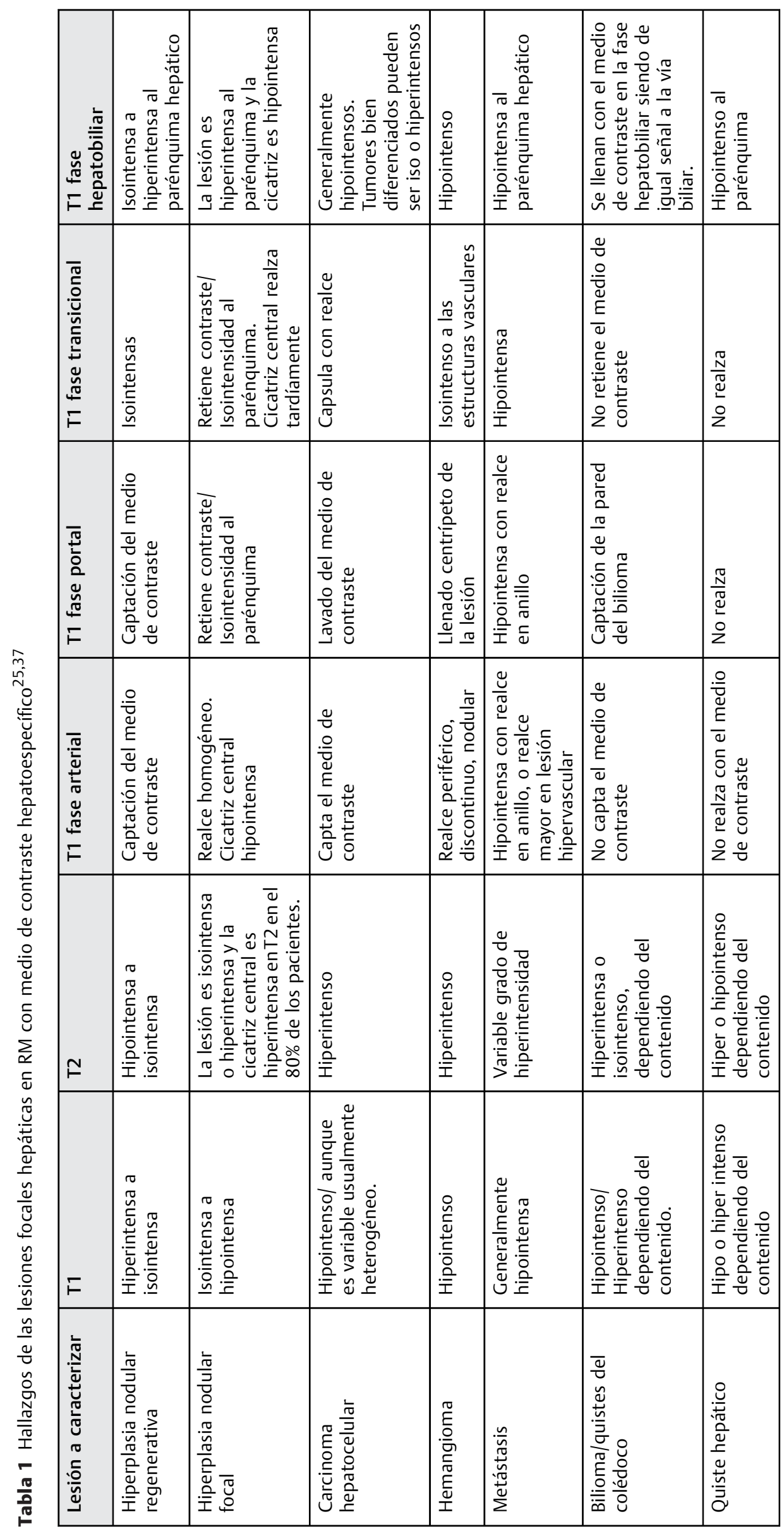

Revista Argentina de Radiología / Argentinian Journal of Radiology Vol. 82 No. 1/2018 
obstrucciones y estenosis. Además, brinda una mayor detección de la filtración biliar y caracteriza mejor las lesiones quísticas que comunican con la vía biliar. ${ }^{15,17,25,49-52}$

La evaluación prequirúrgica con agentes hepatoespecíficos, ha demostrado que la disminución del realce parenquimatoso relativo en la fase hepatobiliar constituye un factor de riesgo independiente para el desarrollo de falla hepática posterior a la resección hepática mayor. ${ }^{23}$

\section{Hiperplasia Nodular Regenerativa}

Este tipo de lesiones han sido descritas en hígados cirróticos, siendo macroscópicamente y microscópicamente similares a una HNF. Se ha planteado su posible etiología como una respuesta hiperplásica del tejido a una noxa vascular en el contexto de pacientes con síndrome de Budd-Chiari, trombosis portal, entre otras. Sus características por imagen son múltiples lesiones hiperintensas a isointensas en secuencias ponderadas en $\mathrm{T} 1$, hipointensas a isointensas en secuencias ponderadas en $\mathrm{T} 2$, y en las secuencias dinámicas contrastadas presentan un realce en la fase arterial y una retención del contraste en la fase portal y transicional. En la fase hepatobiliar demuestran la retención del ácido gadoxético ${ }^{6}$ (-Fig. 3 ).

\section{Hiperplasia Nodular Focal y Adenomas Hepatocelulares}

La HNF es típicamente asintomática, encontrada en mujeres jóvenes de edad media y es el segundo tumor benigno más común después del hemangioma. La literatura reporta una prevalencia hasta del 8\%; y el 70\% de los pacientes presenta una sola lesión. ${ }^{53}$

En la HNF existe una captación homogénea del medio de contraste en la fase arterial, que se conserva durante la fase portal y transicional. En la fase hepatobiliar son isointensas o hiperintensas respecto al parénquima hepático por la retención del contraste. Ese tipo de lesiones, especialmente las de mayor tamaño presentan una cicatriz central hasta en
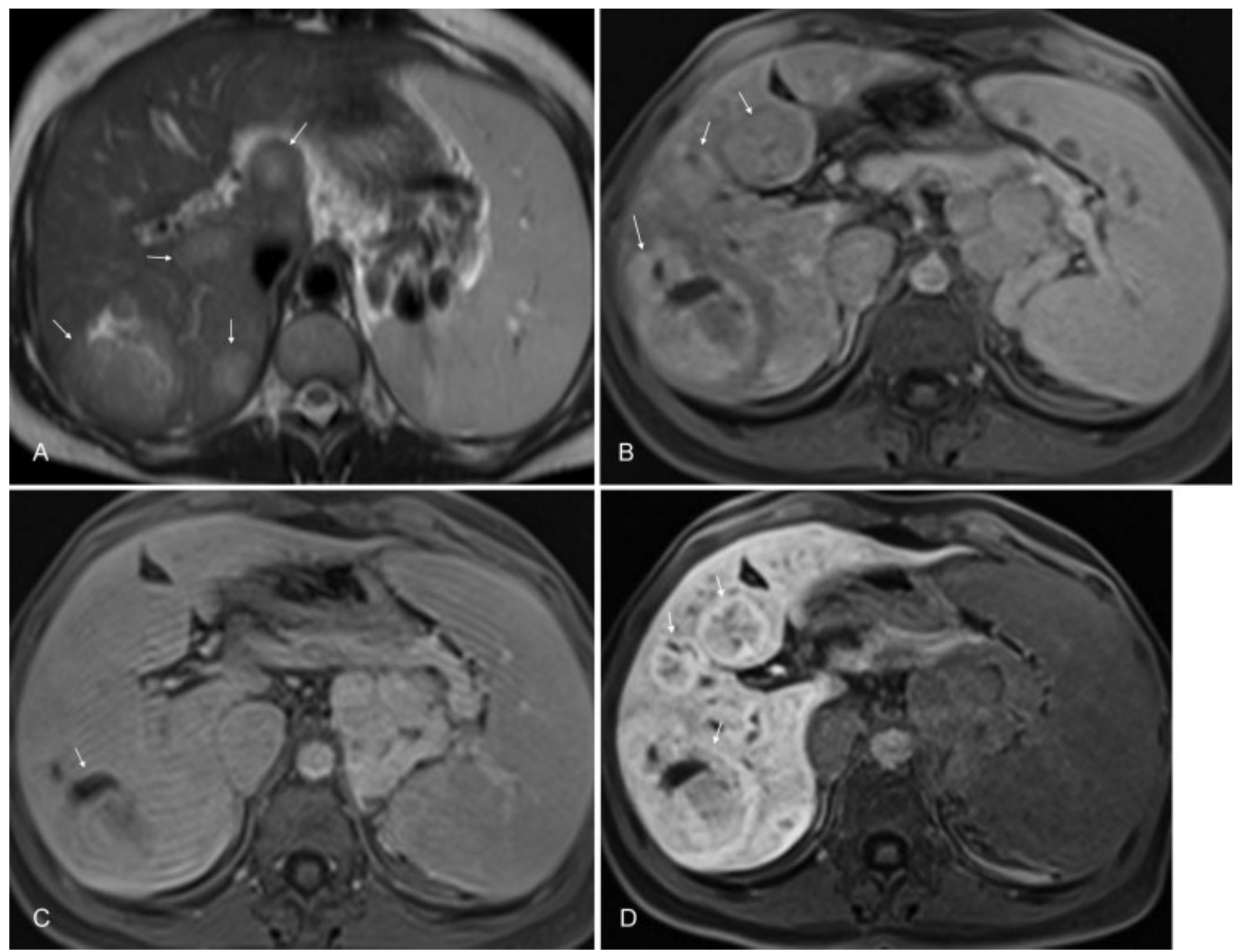

Fig. 3 Paciente de 25 años de edad, con fibrosis hepática congénita y trombosis portal. Se realizó estudio de RM con agente hepatoespecífico. Hiperplasia nodular regenerativa (flechas). Secuencias ponderadas en T2 axial sin saturación grasa en la que se observaron lesiones hepáticas múltiples con señal intermedia (A). Secuencias ponderadas en T1 VIBE (volumetric interpolated breath-hold examination) con saturación grasa en la fase arterial en la que se observa la hipervascularidad de las lesiones (B). Secuencias ponderadas en T1 VIBE con saturación grasa en la fase portal (C), se observó la isointensidad de las lesiones con la lesión dominante localizada en los segmentos VI-VII con zona quística central. Secuencias ponderadas en T1 VIBE con saturación grasa en la fase hepatobiliar (D), en la que se demuestran las lesiones con retención del medio de contraste, siendo isointensas a hiperintensas al parénquima hepático. Adicionalmente se observó degeneración cavernomatosa de la porta con múltiples colaterales en el hilio esplénico como signos de hipertensión portal. 
el $80 \%$ de los casos. Su apariencia típica por RM es de alta señal en secuencias ponderadas en $\mathrm{T} 2 \mathrm{y}$ de baja señal en secuencias ponderadas en $\mathrm{T} 1$, su comportamiento con el medio de contraste es de baja señal en la fase arterial, con realce gradual progresivo en las fases tardías. ${ }^{6}$ Sin embargo, con el uso de ácido gadoxético, la cicatriz central de la HNF puede permanecer hipointensa respecto al resto de la lesión, la cual presenta retención del contraste por los hepatocitos al interior de la lesión. ${ }^{25,37}$ La combinación de realce intenso en la fase arterial y ligero realce en la fase hepatobiliar ha demostrado una sensibilidad de $83 \%$ y una especificidad de $98 \%^{43}$ (-Fig. 4).
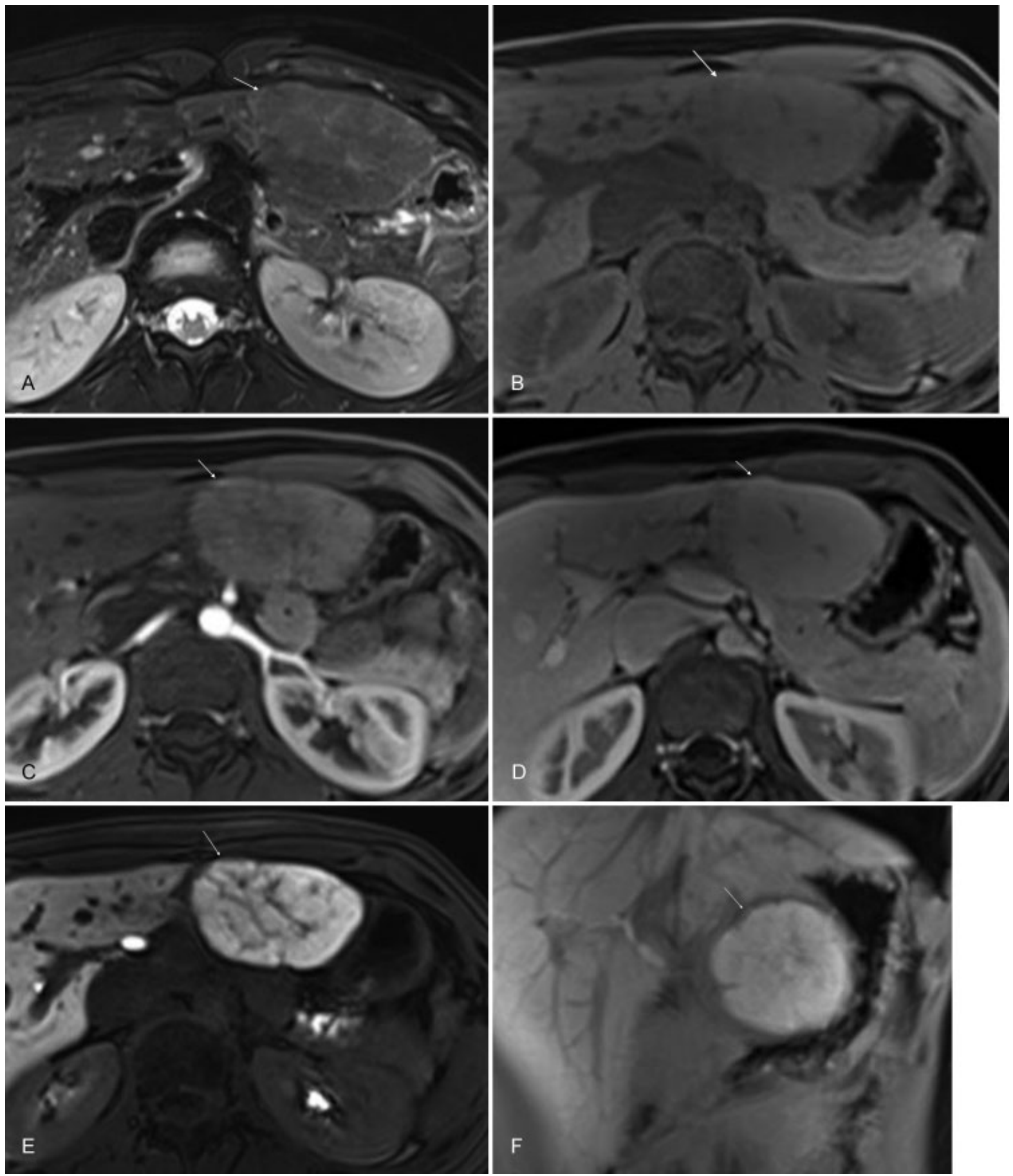

Fig. 4 Paciente femenina de 17 años, con hallazgo incidental de lesión focal visualizada por ecografía en el lóbulo hepático izquierdo, se solicitó RM para caracterización. Hiperplasia nodular focal (flechas). Secuencia ponderada en T2 axial con saturación grasa (A), se observó lesión focal hipointensa con un área central de hiperintensidad correspondiente a la cicatriz central. Secuencia ponderada en T1 VIBE con saturación grasa sin medio de contraste (B), se observó la lesión isointensa con la cicatriz central hipointensa. Secuencia ponderada en T1 VIBE con saturación grasa con medio de contraste en fase arterial (C), se observó hiperintensidad de la lesión con realce intenso y homogéneo. Secuencia ponderada en T1 VIBE con saturación grasa en fase portal (D), se observó isointensidad de la lesión por retención del medio de contraste sin realce de la cicatriz. Secuencia ponderada en T1 con saturación grasa en fase hepatobiliar, axial (E) y coronal (F), en las que se pudo ver hiperintensidad de la lesión por la excreción hepatobiliar del medio de contraste con la cicatriz central hipointensa. 
Los AHC son neoplasias benignas hepáticas monoclonales infrecuentes, compuestas por células que simulan hepatocitos normales. Se presentan principalmente en mujeres usuarias de anticonceptivos orales (ACO) por más de dos años. Desde el punto de vista genético y patológico, se clasifican en cuatro categorías: en primer lugar, el adenoma hepatocelular inflamatorio; en segundo lugar, los adenomas asociados con la mutación del factor nuclear hepatocítico (FNH) $-1 \alpha$; en tercer lugar, los adenomas asociados con la mutación del gen que codifica para la $\beta$-catenina y por último los adenomas no clasificados. ${ }^{33}$ Su comportamiento en RM es variable en las diferentes secuencias pero por lo general, son lesiones hiperintensas en secuencias ponderadas en T2, hipointensas en secuencias ponderadas en T1, excepto en la variedad esteatósica que pueden ser isointensos a hiperintensos en secuencias ponderadas en T1 y T2, con caída de señal en la secuencia ponderada en T1 fuera de fase. Son en su mayoría hipervasculares, y sin retención del medio de contraste en la fase hepatobiliar., ${ }^{6,33}$ Esas lesiones contienen hepatocitos pero son carentes de conductos biliares, lo que impide la excreción del contraste en la fase hepatobiliar. Ese es el hallazgo determinante para diferenciar la $\mathrm{HNF}$ del $\mathrm{AHC}^{6}$ (- Fig. 5).

Es importante diferenciar las lesiones focales hepáticas benignas, especialmente en el debate diagnóstico entre HNF y AHC, puesto que su manejo difiere significativamente. La primera se realiza con manejo conservador, y la segunda de acuerdo a criterios establecidos, llegando a requerir resección quirúrgica dada la posibilidad de rotura o transformación maligna. ${ }^{54}$

\section{Carcinoma Hepatocelular}

El CHC es la quinta causa de cáncer a nivel mundial y es la segunda causa de muerte por cáncer cada año. La detección de un CHC sintomático da un pronóstico de $0-10 \%$ de sobrevida a 5 años. ${ }^{55}$ Por otra parte, los CHC tempranos pueden ser curados, dependiendo de su estado, de acuerdo
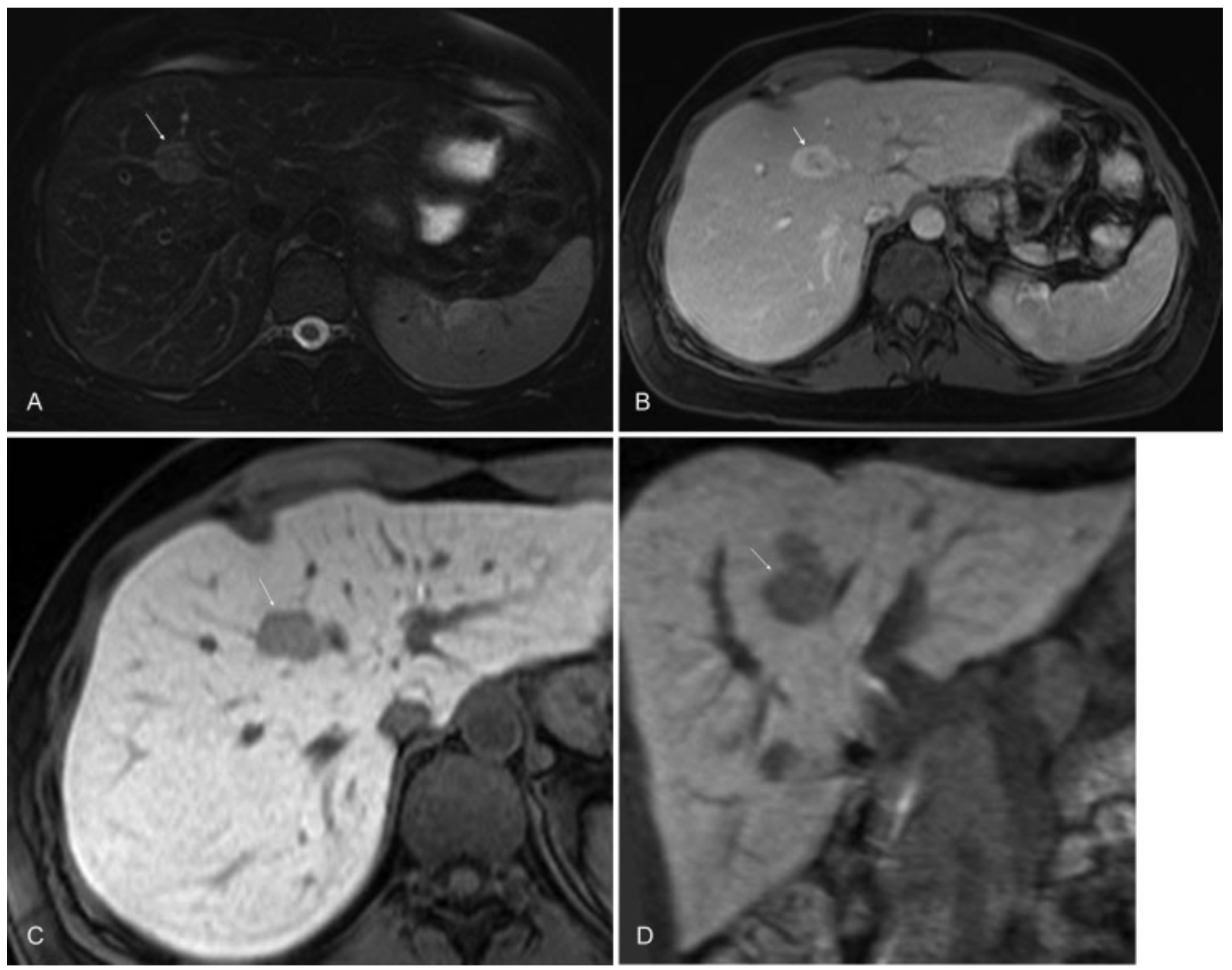

Fig. 5 Paciente femenina de 25 años, con antecedente de consumo de anovulatorios orales, hallazgo incidental de lesión focal visualizada por ecografía que fue realizada por dolor abdominal, se solicitó RM para caracterización. Adenoma hepatocelular (flechas). Estudio de RM. Secuencias ponderadas en T2 axial con saturación grasa (A) se observó lesión focal con señal intermedia en el segmento IVA. Secuencia ponderada en T1 VIBE con saturación grasa con medio de contraste órgano específico en fase portal (B) se observó hiperintensidad de la lesión con realce heterogéneo. Secuencia ponderada en T1 con saturación grasa en fase hepatobiliar, axial (C) y coronal (D) en las que se observó hipointensidad de la lesión sobre un parénquima hepático con alta intensidad de señal. 
con diferentes métodos que incluyen: terapia con ablación, manejo con resección tumoral y trasplante hepático. ${ }^{56}$ La sensibilidad y especificidad para la detección del CHC en los estudios de RM con medio de contraste convencional es entre 82-99\% y 70-95\% respectivamente. ${ }^{29}$ Sin embargo existen espectros de $\mathrm{CHC}$ que no pueden ser adecuadamente caracterizados con los medios de contraste extracelulares, en ese caso puede ser útil el uso del ácido gadoxético especialmente en las lesiones con pobre expresión de transportadores OATP y MRP. Ese hecho se produce a medida que la lesión empeora el grado de diferenciación, demostrando en imágenes de RM baja intensidad de señal en la fase hepatobiliar., ${ }^{2,3}$ En el caso de algunos nódulos displásicos de alto grado y en los $\mathrm{CHC}$ bien diferenciados (2-8\% de los $\mathrm{CHC}$ ), que tienen mayor expresión de los transportadores, se puede observar algún grado de realce en la fase hepatobiliar ${ }^{6}$ (-Fig. 6).

\section{Hemangioma}

Los hemangiomas son el tumor hepático benigno más común del hígado. Presenta diferentes patrones de contraste. Los hemangiomas típicos muestran un realce periférico, discontinuo y nodular en la fase arterial, que aumenta progresivamente hasta el llenado completo en las fases tardías. En la fase hepatobiliar su intensidad de señal es menor respecto al parénquima hepático. ${ }^{36,57}$

Su apariencia puede ser diferente en las fases dinámicas con agentes hepatoespecíficos, comparado con los contrastes extracelulares. Debido a la rápida captación del ácido gadoxético por el parénquima hepático, los hemangiomas pueden hacer un pseudo-lavado y tornarse hipointensos en la fase transicional. ${ }^{6}$ Como fue observado por Don y col., ${ }^{6}$ los hemangiomas de llenado rápido con fenómeno de pseudolavado posterior a la administración de ácido gadoxético
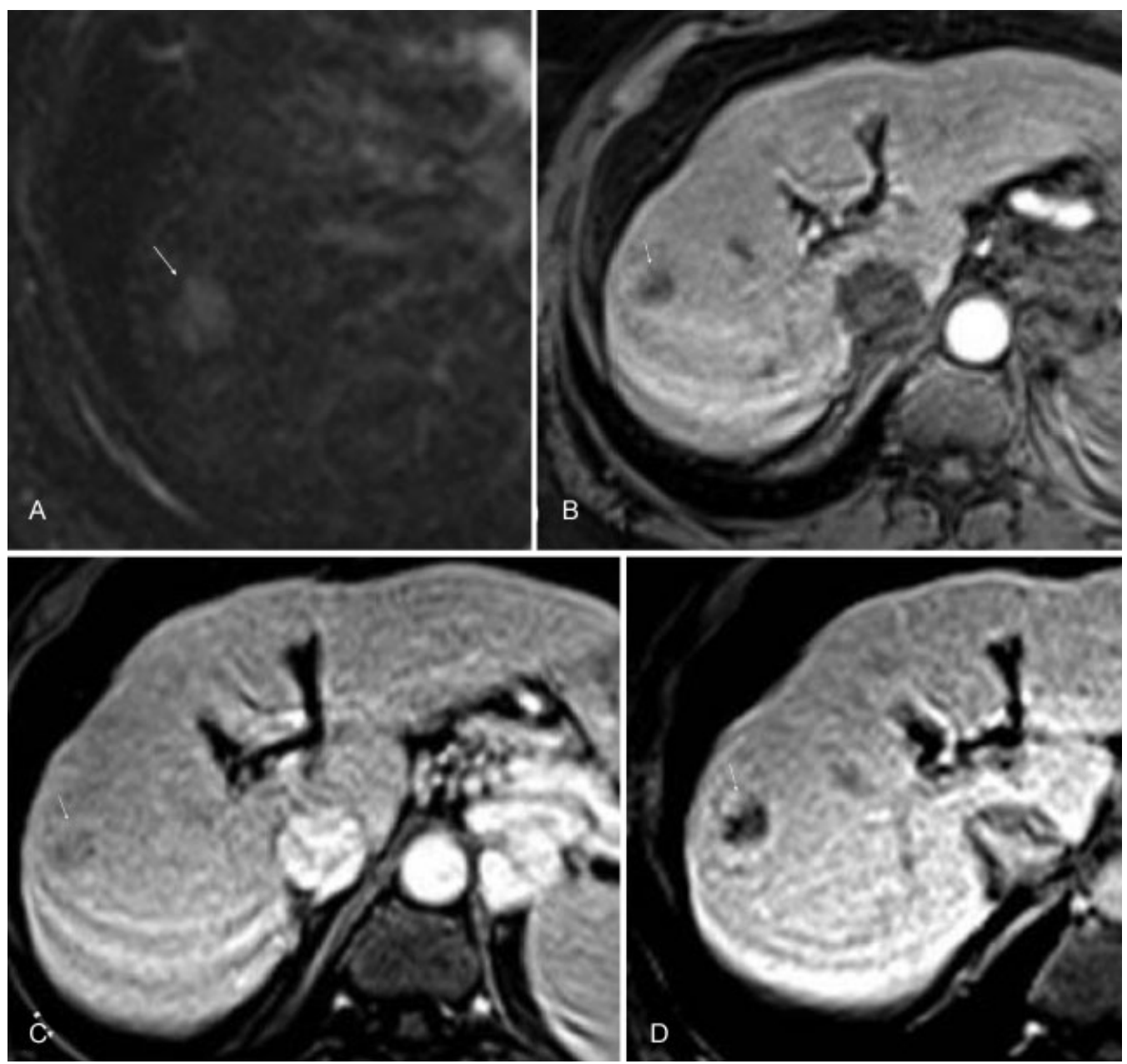

Fig. 6 Paciente masculino 60 años, con cirrosis criptogénica, Child A, con lesión focal hepática (flechas), que no reunía los criterios de CHC, por lo que se le realizó estudio con medio de contraste hepato específico para caracterización. Secuencia ponderada en T2 axial con saturación grasa (A), se observó una lesión focal con señal intermedia localizada en el segmento VIII. Secuencia ponderada en T1 VIBE con saturación grasa con medio de contraste en fase arterial (B), se observó la lesión hipovascular. Secuencia ponderada en T1 VIBE con saturación grasa con medio de contraste en fase portal (C), se observó la lesión hipovascular con formación de capsula. Secuencia ponderada en T1 VIBE con saturación grasa con medio de contraste en fase hepatobiliar (D), se observó la lesión hipointensa con respecto al parénquima hepático, indicando la ausencia de hepatocitos funcionales para la excreción hepatobiliar, comportamiento que en el contexto de cirrosis hepática corresponde a un foco de CHC hipovascular comprobado por medio de análisis histológico. 
pueden confundirse con metástasis hipervasculares, sin embargo el análisis detallado del realce puede hacer el diagnóstico diferencial en esos casos. En las metástasis se observa lavado más rápido que en las estructuras vasculares, a diferencia de los hemangiomas que lavan de forma paralela a los vasos. ${ }^{58}$ Adicionalmente la mayor intensidad de señal en secuencias ponderadas en $\mathrm{T} 2$ de los hemangiomas indica su diagnóstico ${ }^{6}$ (-Fig. 7).

\section{Metástasis}

El principal objetivo de la RM en el hígado y en la enfermedad metastásica es identificar las lesiones resecables y potencialmente curables. ${ }^{37}$ Las lesiones metastásicas no contienen hepatocitos, por lo tanto se van a presentar hipointensas en la fase hepatobiliar, independientemente de si son hipervasculares o hipovasculares. ${ }^{25}$
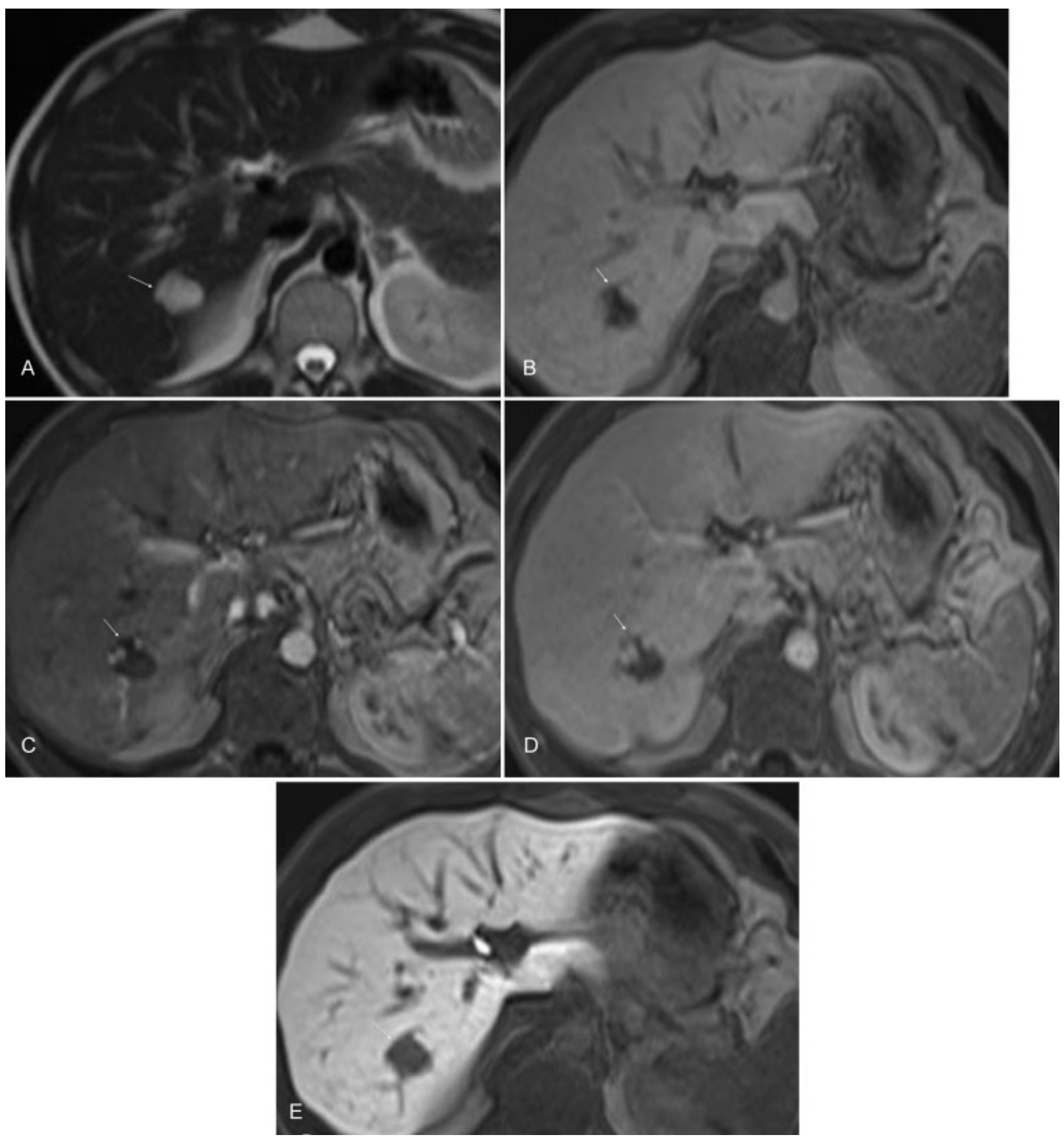

Fig. 7 Paciente masculino de 33 años de edad, hallazgo incidental de lesión hepática. Hemangioma (flechas). Estudio de RM. Secuencia ponderada en T2 HASTE donde se observó lesión focal hiperintensa en el segmento VII (A), secuencia ponderada en T1 VIBE sin medio de contraste (B), se observó la lesión hipointensa. Secuencia ponderada en T1 VIBE con saturación grasa con medio de contraste en fase arterial (C) y fase portal (D). Se observó la lesión con realce globular, periférico y discontinuo. Secuencia ponderada en T1 VIBE con saturación grasa con medio de contraste en fase de excreción hepatobiliar (E), se observó la lesión isointensa a las estructuras vasculares e hipointensa al parénquima hepático. 

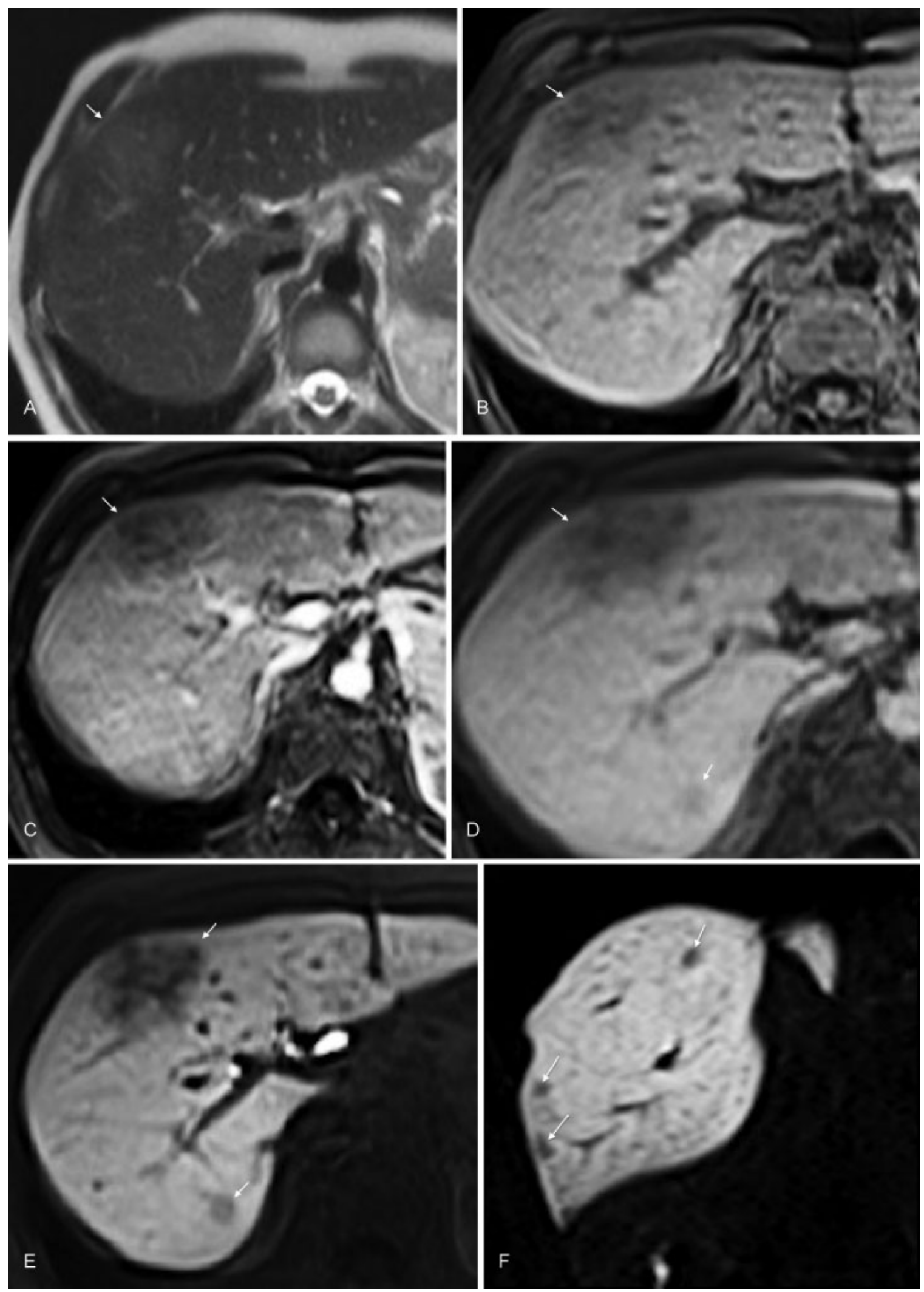

Fig. 8 Paciente femenina de 36 años de edad, con tumor carcinoide. Metástasis hepáticas (flechas). Estudio de RM. Secuencia ponderada en T2 HASTE axial (A), pudo notarse una lesión focal en segmento IVA y con señal intermedia. Secuencia ponderada en T1 axial VIBE con saturación grasa sin medio de contraste (B), se observó una lesión hipointensa a isointensa. Secuencia ponderada en T1 axial VIBE con saturación grasa luego de la administración de medio de contraste: fase arterial (C) y fase portal (D). Se pudo observar la hipovascularidad de la lesión siendo hipointensa en ambas secuencias. Secuencia ponderada en T1 axial VIBE con saturación grasa luego de la administración de medio de contraste en fase hepatobiliar, imagen axial (E) e imagen coronal (F), se observó la lesión hipointensa, observando además múltiples lesiones adicionales no visualizadas en las otras secuencias (flechas). Los hallazgos tuvieron comprobación histológica de metástasis por carcinoide. 
En los pacientes con quimioterapia, puede ser difícil visualizar las metástasis, debido a cambios del parénquima hepático, por el síndrome de oclusión sinusoidal y por la presencia de esteatosis hepática que sigue al tratamiento, lo que dificulta su visualización y caracterización en la tomografía. ${ }^{43}$

Es clara la importancia al elegir un método altamente sensible para la identificación de las metástasis y cobra relevancia en el contexto del cáncer de colon, donde se puede observar que la adecuada identificación del compromiso hepático secundario será definitivo para la elección del tratamiento y constituye la principal causa de recaída tumoral. Chen y col., ${ }^{59}$ realizaron un metaanálisis, en el cual se concluyó que la sensibilidad y especificidad de la RM con ácido gadoxético fue de 93 y 95\% respectivamente para la detección de metástasis. ${ }^{59}$ Adicionalmente, el ácido gadoxético ha demostrado ser superior a otras modalidades por imagen para la detección de metástasis hepáticas menores a $1 \mathrm{~cm}^{13,42-45}$ (- Fig. 8).

\section{Quiste hepático vs bilioma}

Luego de los procedimientos quirúrgicos, la colangiografía por RM puede ayudar a caracterizar mejor las colecciones y hacer un diagnóstico diferencial adecuado entre biliomas y otras lesiones quísticas. Para el caso del bilioma se define en la fase hepatobiliar la comunicación de la colección con el árbol biliar. ${ }^{17}$

Para optimizar el uso del medio de contraste, es indispensable conocer la función hepática del paciente, indicado por la concentración de bilirrubina. Cuando es mayor de $3 \mathrm{mg} / \mathrm{dl}$, la excreción del medio de contraste se altera, dado que el ácido gadoxético actúa como un antagonista competitivo de ella. Se considera excreción normal cuando el contraste se observa en la ampolla de Váter a los 20 - 30 minutos y se encuentra retardado cuando se da entre 30 y 60 minutos $^{17}$ (-Fig. 9).
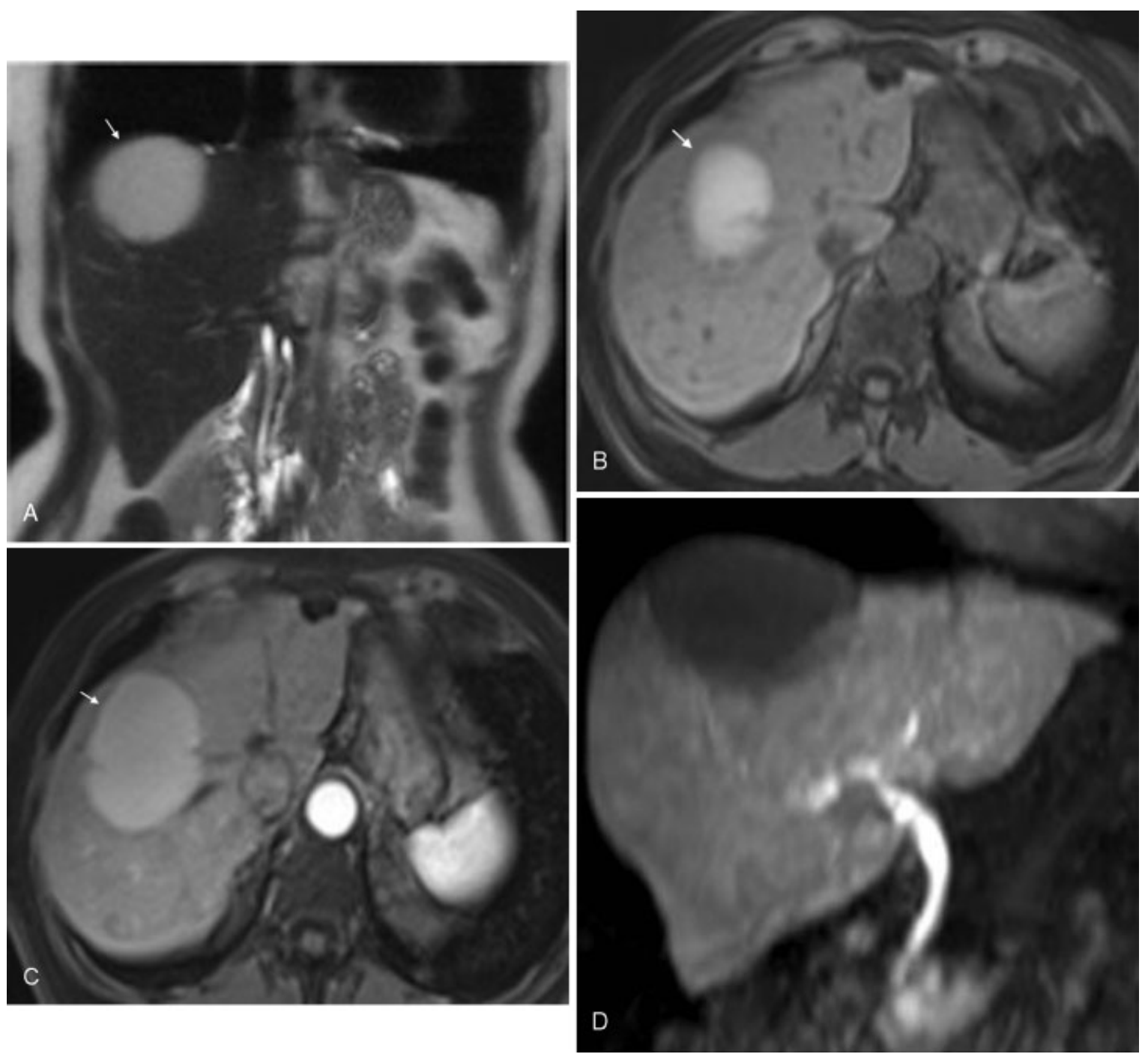

Fig. 9 Paciente femenina de 86 años de edad, antecedente de colecistectomía a quien se le realizó una tomografía contrastada, donde se observó una imagen quística subdiafragmática (flechas). Se le realizó RM para descartar filtración biliar y formación de bilioma. Secuencia ponderada en T2 HASTE coronal (A). Secuencia ponderada en T1 VIBE con saturación grasa fase simple (B), secuencia ponderada en T1 VIBE con saturación grasa fase arterial (C). Secuencia ponderada en T1 VIBE coronal con saturación grasa en fase hepatobiliar (D), en la cual se observa excreción del contraste a la vía biliar normal (flecha). Los hallazgos descartan filtración biliar y formación de bilioma. 

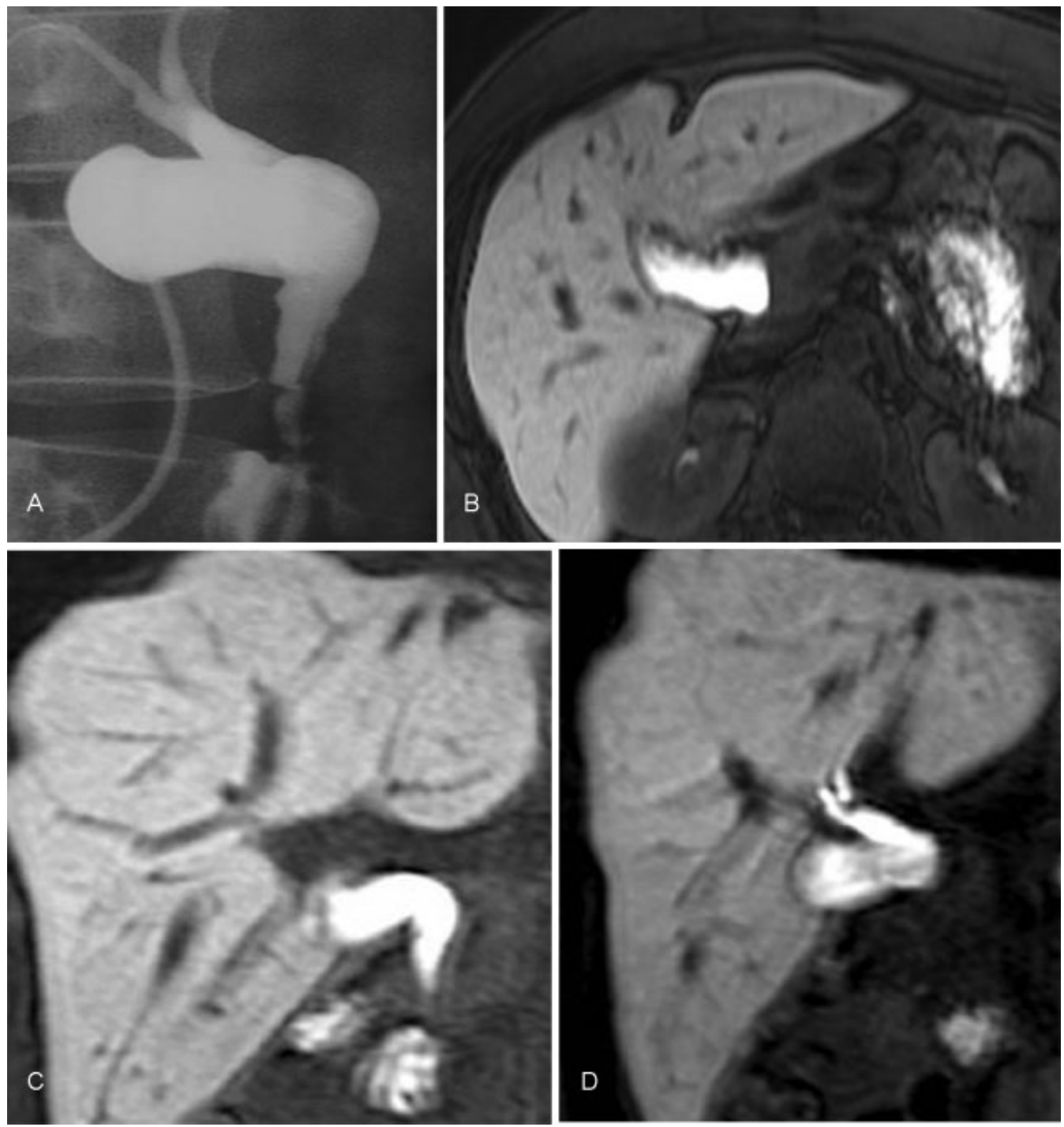

Fig. 10 Paciente femenina de 56 años de edad, antecedente de colecistectomía y sospecha de quiste del colédoco por ecografía. Colangiografía por tubo en T (A), donde se observó un quiste sacular del colédoco sin determinar adecuadamente el cuello del quiste. Secuencia ponderada T1 con saturación grasa en fase hepatobiliar axial (B), coronal (C y D) en las cuales se observó divertículo del colédoco con llenado progresivo por contraste, correspondiendo a quiste del colédoco Todani II.

\section{Quistes del colédoco}

En la evaluación de variantes congénitas como los quistes del colédoco, las imágenes en fase hepatobiliar son de gran ayuda, especialmente en el tipo II, IV y V para establecer su comunicación con el árbol biliar, permitiendo diferenciar estos quistes de los demás quistes hepáticos que no se comunican con la vía biliar y, por lo tanto, no van a requerir a futuro un seguimiento o exámenes adicionales ${ }^{16}$ (- Fig. 10).

En la - Tabla 2 se describe la clasificación de Todani de los quistes del colédoco.

\section{Fístula Biliar}

La RM es un herramienta útil, no invasiva, para la detección de complicaciones posquirúrgicas en el árbol biliar, como son las fistulas, las estenosis o la ligadura de la vía biliar inadvertida. ${ }^{25}$ Durante la fase hepatobiliar se permite visualizar adecuadamente la anatomía pre y posquirúrgica de la vía biliar, incluyendo las complicaciones poscolecistectomía. Castellanos y col., ${ }^{60}$ detectaron durante la fase hepatobiliar con ácido gadoxético el $100 \%$ de las fístulas biliares, luego de 20 minutos de la administración del medio de contraste ${ }^{60}$ ( - Fig. 11).

Tabla 2 Clasificación de Todani de quistes del colédoco ${ }^{16}$

\begin{tabular}{|l|l|}
\hline Tipo & Hallazgos \\
\hline I & $\begin{array}{l}\text { Dilatación fusiforme/sacular de la vía biliar } \\
\text { extra hepática }\end{array}$ \\
\hline II & Divertículo de la vía biliar extra hepática \\
\hline III & Coledococele \\
\hline IV & $\begin{array}{l}\text { Dilataciones de la vía biliar intra y extra } \\
\text { hepática }\end{array}$ \\
\hline V & $\begin{array}{l}\text { Múltiples dilataciones quísticas intrahepáticas } \\
\text { (enfermedad de Caroli) }\end{array}$ \\
\hline
\end{tabular}



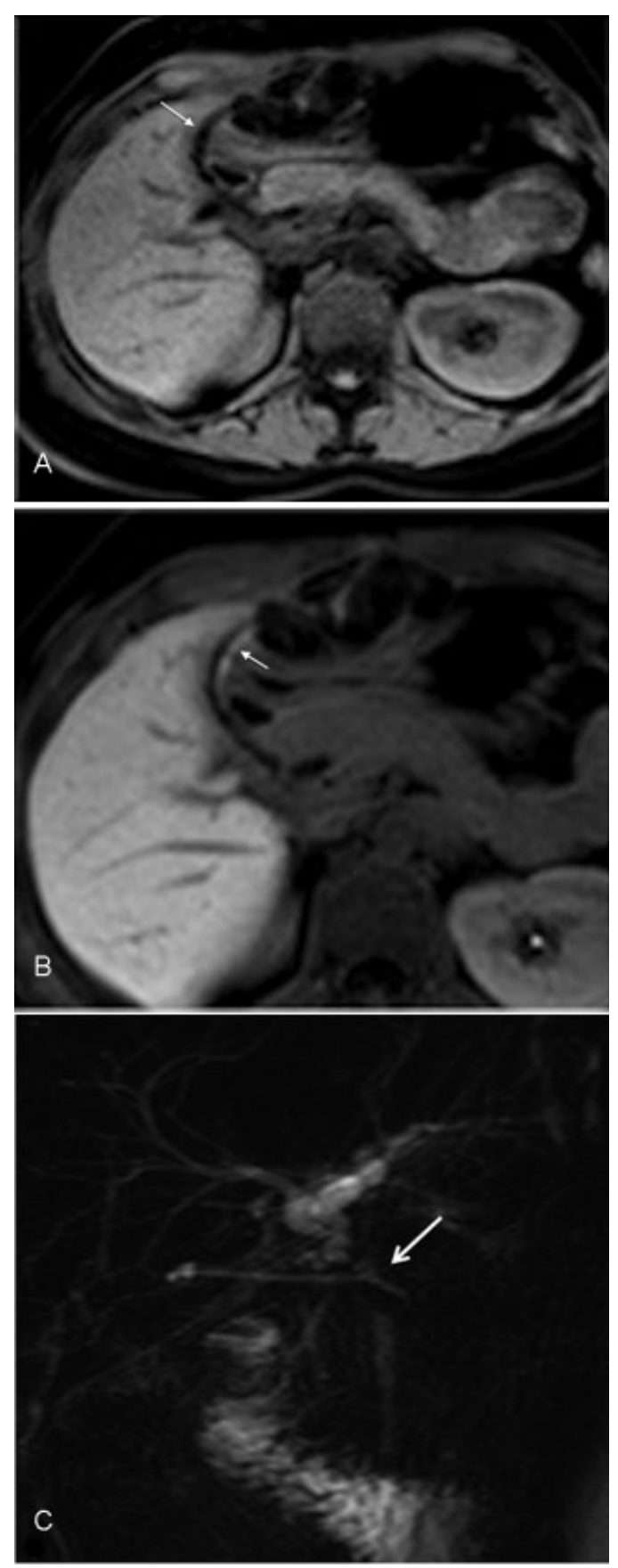

Fig. 11 Paciente femenina de 40 años, se realizó colecistectomía abierta por colecistitis y se dejó drenaje anterior a cavidad abdominal y un tubo en T. Se sospechó de una filtración. La fistulografía convencional no demostró fístulas. Se realizó un estudio de RM. Secuencia ponderada en T1 axial VIBE con saturación grasa en fase simple (A). Se observó un catéter de abordaje anterior con ubicación distal en el hilio hepático (flecha). Secuencia ponderada en T1 axial VIBE con saturación grasa con medio de contraste en fase hepatobiliar adquirida a los 20 minutos (B), se pudo identificar un medio de contraste extraluminal adyacente al catéter (flecha). Secuencias de colangio RM de corte único (C), se observó solución de continuidad de la vía biliar extrahepática entre el conducto hepático común proximal y el colédoco con un defecto de $13 \mathrm{~mm}$, identificando un remanente del hepático común de aproximadamente $7 \mathrm{~mm}$, configurando una lesión Bismuth II. El tubo en T tiene el extremo proximal y distal por fuera de la vía biliar (flecha). Los hallazgos tuvieron confirmación quirúrgica.

\section{Conclusión}

Los medios de contraste hepatoespecíficos son una herramienta valiosa para la evaluación complementaria de las lesiones focales hepáticas, especialmente en aquellas con un comportamiento no concluyente en las fases dinámicas contrastadas; al igual que en el diagnóstico diferencial de HNF y AHC, son útiles también para la detección de metástasis menores a $1 \mathrm{~cm}$, en términos de pronóstico y manejo del paciente oncológico. Con respecto a la valoración complementaria de la vía biliar, este tipo de agentes permiten definir la presencia de lesiones traumáticas del árbol biliar y también brindan la posibilidad de establecer la comunicación de algunas lesiones quísticas con la vía biliar. En ese contexto, los medios de contraste hepatoespecíficos, parecen tener un futuro muy prometedor para su uso rutinario en la RM hepática.

\section{Agradecimientos}

Al proyecto de sostenibilidad de la vicerrectoría de Investigación de la Universidad de Antioquia, Medellín, Colombia.

\section{Responsabilidades éticas}

Protección de personas y animales. Los autores declaran que para esta investigación no se han realizado experimentos en seres humanos ni en animales.

Confidencialidad de los datos. Los autores declaran que han seguido los protocolos de su centro de trabajo sobre la publicación de datos de pacientes.

Derecho a la privacidad y consentimiento informado. Los autores declaran que en este artículo no aparecen datos de pacientes.

\section{Conflicto de intereses}

Los autores declaran no tener ningún conflicto de intereses.

\section{Bibliografía}

1 Van Beers BE, Pastor CM, Hussain HK. Primovist, Eovist: what to expect? J Hepatol 2012;57(02):421-429

2 Choi J-Y, Lee JM, Sirlin CBCT. CT and MR imaging diagnosis and staging of hepatocellular carcinoma: part I. Development, growth, and spread: key pathologic and imaging aspects. Radiology 2014; 272(03):635-654

3 Choi J-Y, Lee JM, Sirlin CBCT. CT and MR imaging diagnosis and staging of hepatocellular carcinoma: part II. Extracellular agents, hepatobiliary agents, and ancillary imaging features. Radiology 2014;273(01):30-50

4 Carrasco Muñoz S, Calles Blanco C, Marcin J, Fernández Álvarez C, Lafuente Martínez J. Contrastes basados en gadolinio utilizados en resonancia magnética. Radiologia 2014;56(Suppl 1):21-28

5 Guglielmo FF, Mitchell DG, Gupta S. Gadolinium contrast agent selection and optimal use for body MR imaging. Radiol Clin North Am 2014;52(04):637-656

6 Campos JT, Sirlin CB, Choi JY. Focal hepatic lesions in Gd-EOBDTPA enhanced MRI: the atlas. Insights Imaging 2012;3(05):451-474 
7 Morana G, Salviato E, Guarise A. Contrast agents for hepatic MRI. Cancer Imaging 2007;7 Spec No A:S24-S27

8 Fischer MA, Raptis DA, Donati OF, et al. MR imaging features for improved diagnosis of hepatocellular carcinoma in the noncirrhotic liver: Multi-center evaluation. Eur J Radiol 2015;84 (10):1879-1887

9 Raman SS, Leary C, Bluemke DA, et al; United States EOB Study Group. Improved characterization of focal liver lesions with liverspecific gadoxetic acid disodium-enhanced magnetic resonance imaging: a multicenter phase 3 clinical trial. J Comput Assist Tomogr 2010;34(02):163-172

10 Sutherland T, Seale M, Yap K. Part 1: MRI features of focal nodular hyperplasia with an emphasis on hepatobiliary contrast agents. J Med Imaging Radiat Oncol 2014;58(01):50-55

11 Sutherland T, Seale M, Yap K. Part 2: MRI of hypervascular focal liver lesions using liver specific contrast agents. J Med Imaging Radiat Oncol 2014;58(01):56-63

12 Joo I, Lee JM. Recent Advances in the Imaging Diagnosis of Hepatocellular Carcinoma: Value of Gadoxetic Acid-Enhanced MRI. Liver Cancer 2016;5(01):67-87

13 Shimada K, Isoda H, Hirokawa Y, Arizono S, Shibata T, Togashi K. Comparison of gadolinium-EOB-DTPA-enhanced and diffusionweighted liver MRI for detection of small hepatic metastases. Eur Radiol 2010;20(11):2690-2698

14 Lee NK, Kim S, Lee JW, et al. Biliary MR imaging with Gd-EOBDTPA and its clinical applications. Radiographics 2009;29(06): 1707-1724

15 Tamada T, Ito K, Yasokawa K, et al. Accumulation of Bile in the Gallbladder: Evaluation by means of Serial Dynamic ContrastEnhanced Magnetic Resonance Cholangiography with Gadolinium Ethoxybenzyl Diethylenetriaminepentaacetic Acid. Gastroenterol Res Pract 2014;2014:479067

16 Gupta RT, Brady CM, Lotz J, Boll DT, Merkle EM. Dynamic MR imaging of the biliary system using hepatocyte-specific contrast agents. AJR Am J Roentgenol 2010;195(02):405-413

17 Gupta RT. Evaluation of the biliary tree and gallbladder with hepatocellular MR contrast agents. Curr Probl Diagn Radiol 2013; 42(02):67-76

18 Reimer P, Schneider G, Schima W. Hepatobiliary contrast agents for contrast-enhanced MRI of the liver: properties, clinical development and applications. Eur Radiol 2004;14 (04):559-578

19 Thian YL, Riddell AM, Koh DM. Liver-specific agents for contrastenhanced MRI: role in oncological imaging. Cancer Imaging 2013; 13(04):567-579

20 Kitao A, Zen Y, Matsui O, et al. Hepatocellular carcinoma: signal intensity at gadoxetic acid-enhanced MR Imaging-correlation with molecular transporters and histopathologic features. Radiology 2010;256(03):817-826

21 Tsuda N, Matsui O. Cirrhotic rat liver: reference to transporter activity and morphologic changes in bile canaliculi-gadoxetic acid-enhanced MR imaging. Radiology 2010;256(03):767-773

$22 \mathrm{Kim}$ JY, Lee SS, Byun JH, et al. Biologic factors affecting HCC conspicuity in hepatobiliary phase imaging with liver-specific contrast agents. AJR Am J Roentgenol 2013;201(02):322-331

23 Wibmer A, Prusa AM, Nolz R, Gruenberger T, Schindl M, BaSsalamah A. Liver failure after major liver resection: risk assessment by using preoperative Gadoxetic acid-enhanced 3-T MR imaging. Radiology 2013;269(03):777-786

24 Motosugi U, Ichikawa $\mathrm{T}$, Sou $\mathrm{H}$, et al. Liver parenchymal enhancement of hepatocyte-phase images in Gd-EOB-DTPAenhanced MR imaging: which biological markers of the liver function affect the enhancement? J Magn Reson Imaging 2009; 30(05):1042-1046

25 Seale MK, Catalano OA, Saini S, Hahn PF, Sahani DV. Hepatobiliaryspecific MR contrast agents: role in imaging the liver and biliary tree. Radiographics 2009;29(06):1725-1748
26 Frydrychowicz A, Lubner MG, Brown JJ, et al. Hepatobiliary MR imaging with gadolinium-based contrast agents. J Magn Reson Imaging 2012;35(03):492-511

27 Liu PS. Liver Mass Evaluation in Patients Without Cirrhosis: A Technique-Based Method. Radiol Clin North Am 2015;53(05): 903-918

28 Gandhi SN, Brown MA, Wong JG, Aguirre DA, Sirlin CB. MR contrast agents for liver imaging: what, when, how. Radiographics 2006;26(06):1621-1636

29 Cossio-Torrico PE, Ramírez-Carmona CR, Stoopen-Rometti M, Perochena-González A, Sosa-Lozano LA, Kimura-Hayama E. Liverspecific gadoxetic acid-enhanced magnetic resonance for focal lesion evaluation. Rev Gastroenterol Mex 2015;80(04):267-275

30 Hamm B, Staks T, Mühler A, et al. Phase I clinical evaluation of Gd-EOBDTPA as a hepatobiliary MR contrast agent: safety, pharmacokinetics, and MR imaging. Radiology 1995;195(03):785-792

31 Vogl TJ, Kümmel S, Hammerstingl R, et al. Liver tumors: comparison of MR imaging with Gd-EOB-DTPA and Gd-DTPA. Radiology 1996;200(01):59-67

32 Reimer P, Rummeny EJ, Shamsi K, et al. Phase II clinical evaluation of Gd-EOB-DTPA: dose, safety aspects, and pulse sequence. Radiology 1996;199(01):177-183

33 McInnes MDF, Hibbert RM, Inácio JR, Schieda N. Focal Nodular Hyperplasia and Hepatocellular Adenoma: Accuracy of Gadoxetic Acid-enhanced MR Imaging-A Systematic Review. Radiology 2015;277(02):413-423

34 Roux M, Pigneur F, Calderaro J, et al. Differentiation of focal nodular hyperplasia from hepatocellular adenoma: Role of the quantitative analysis of gadobenate dimeglumine-enhanced hepatobiliary phase MRI. J Magn Reson Imaging 2015;42(05): 1249-1258

35 Reimer P, Rummeny EJ, Daldrup HE, et al. Enhancement characteristics of liver metastases, hepatocellular carcinomas, and hemangiomas with Gd-EOB-DTPA: preliminary results with dynamic MR imaging. Eur Radiol 1997;7(02):275-280

36 Caraiani C-N, Dan M, Fenesan D-I, Badea R. Description of focal liver lesions with Gd-EOB-DTPA enhanced MRI. Clujul Med 2015; 88(04):438-448

37 Burke C, Alexander Grant L, Goh V, Griffin N. The role of hepatocyte-specific contrast agents in hepatobiliary magnetic resonance imaging. Semin Ultrasound CT MR 2013;34(01):44-53

38 van den Esschert JW, van Gulik TM, Phoa SSKS. Imaging modalities for focal nodular hyperplasia and hepatocellular adenoma. Dig Surg 2010;27(01):46-55

39 Grazioli L, Morana G, Kirchin MA, Schneider G. Accurate differentiation of focal nodular hyperplasia from hepatic adenoma at gadobenate dimeglumine-enhanced MR imaging: prospective study. Radiology 2005;236(01):166-177

40 Denecke T, Steffen IG, Agarwal S, et al. Appearance of hepatocellular adenomas on gadoxetic acid-enhanced MRI. Eur Radiol 2012;22(08):1769-1775

41 Thomeer MG, Willemssen FE, Biermann KK, et al. MRI features of inflammatory hepatocellular adenomas on hepatocyte phase imaging with liver-specific contrast agents. J Magn Reson Imaging 2014;39(05):1259-1264

42 Floriani I, Torri V, Rulli E, et al. Performance of imaging modalities in diagnosis of liver metastases from colorectal cancer: a systematic review and meta-analysis. J Magn Reson Imaging 2010;31(01):19-31

43 Jhaveri K, Cleary S, Audet P, et al. Consensus statements from a multidisciplinary expert panel on the utilization and application of a liver-specific MRI contrast agent (gadoxetic acid). AJR Am J Roentgenol 2015;204(03):498-509

44 Tsurusaki M, Sofue K, Murakami T. Current evidence for the diagnostic value of gadoxetic acid-enhanced magnetic resonance imaging for liver metastasis. Hepatol Res 2016;46 (09):853-861 
45 Lee $\mathrm{KH}$, Lee JM, Park JH, et al. MR imaging in patients with suspected liver metastases: value of liver-specific contrast agent gadoxetic acid. Korean J Radiol 2013;14(06):894-904

46 Shimofusa R, Ueda T, KishimotoT, et al. Magnetic resonance imaging of hepatocellular carcinoma: a pictorial review of novel insights into pathophysiological features revealed by magnetic resonance imaging. J Hepatobiliary Pancreat Sci 2010;17(05):583-589

$47 \mathrm{Kim} \mathrm{SH}$, Kim SH, Lee J, et al. Gadoxetic acid-enhanced MRI versus triple-phase MDCT for the preoperative detection of hepatocellular carcinoma. AJR Am J Roentgenol 2009;192(06):1675-1681

48 Tsuboyama T, Onishi H, Kim T, et al. Hepatocellular carcinoma: hepatocyte-selective enhancement at gadoxetic acid-enhanced MR imaging-correlation with expression of sinusoidal and canalicular transporters and bile accumulation. Radiology 2010;255(03):824-833

49 Kang Y, Lee JM, Kim SH, Han JK, Choi BI. Intrahepatic mass-forming cholangiocarcinoma: enhancement patterns on gadoxetic acidenhanced MR images. Radiology 2012;264(03):751-760

50 Kantarcı M, Pirimoglu B, Karabulut N, et al. Non-invasive detection of biliary leaks using Gd-EOB-DTPA-enhanced MR cholangiography: comparison with T2-weighted MR cholangiography. Eur Radiol 2013;23(10):2713-2722

51 Delgado Cordón F, Vizuete Del Río J, Martín-Benítez G, Ripollés González T, Martínez Pérez MJ. Bile duct tumors. Radiologia 2015; 57(02):101-112

52 Dahlström N, Persson A, Albiin N, Smedby O, Brismar TB. Contrastenhanced magnetic resonance cholangiography with Gd-BOPTA and Gd-EOB-DTPA in healthy subjects. Acta Radiol 2007;48(04): 362-368
53 Cheng YC, Chiang CM, Wu CC, Chai JW. Confluent focal nodular hyperplasia mimicking liver cancer: value of liver-specific contrast-enhanced MRI for diagnosis. J Chin Med Assoc 2012;75 (07):355-358

54 Katabathina VS, Menias CO, Shanbhogue AK, Jagirdar J, Paspulati RM, Prasad SR. Genetics and imaging of hepatocellular adenomas: 2011 update. Radiographics 2011;31(06):1529-1543

55 Barr DC, Hussain HK. MR imaging in cirrhosis and hepatocellular carcinoma. Magn Reson Imaging Clin N Am 2014;22(03):315-335

56 Bruix J, Sherman M; American Association for the Study of Liver Diseases. Management of hepatocellular carcinoma: an update. Hepatology 2011;53(03):1020-1022

57 Goshima S, Kanematsu M, Watanabe H, et al. Hepatic hemangioma and metastasis: differentiation with gadoxetate disodium-enhanced 3-T MRI. AJR Am J Roentgenol 2010;195 (04):941-946

58 Doo KW, Lee CH, Choi JW, Lee J, Kim KA, Park CM. "Pseudo washout" sign in high-flow hepatic hemangioma on gadoxetic acid contrast-enhanced MRI mimicking hypervascular tumor. AJR Am J Roentgenol 2009;193(06):W490-6

59 Chen L, Zhang J, Zhang L, et al. Meta-analysis of gadoxetic acid disodium (Gd-EOB-DTPA)-enhanced magnetic resonance imaging for the detection of liver metastases. PLoS One 2012;7 (11):e48681

60 Alegre Castellanos A, Molina Granados JF, Escribano Fernandez J, Gallardo Muñoz I, Triviño Tarradas FdeA. Early phase detection of bile leak after hepatobiliary surgery: value of Gd-EOB-DTPAenhanced MR cholangiography. Abdom Imaging 2012;37(05): 795-802 